\title{
A SeCSe-Pd(II) Pincer Complex as Highly Efficient Catalyst for Allylation of
}

\section{Aldehydes with Allyltributyltin}

Qingwei Yao and Mathew Sheets

Department of Chemistry and Biochemistry, The Michael Faraday Laboratories,

Northern Illinois University,

DeKalb, Illinois 60115

\section{CONTENTS}

General

Synthesis of the SeCSe-Pd(II) Pincer Complex 4

Allylation of Aldehydes with Allyltributyltin in the Presence of the

SeCSe-Pd(II) Pincer Catalyst 4. Representative Procedure

$3 S$

Table S-1. Allylation of Aldehydes with Allyltributyltin 7

Using the Pd-Complex 4 as Catalyst

${ }^{1} \mathrm{H}$ NMR Spectroscopic Study on the Reaction of the Pd Complex 4 with

Allyltributyltin 7: Identification of the Transmetalation Product 9

$5 S$

NMR Spectroscopic data of Compounds 8b-8g

References

${ }^{1}$ H NMR Spectrum of Compound 4

${ }^{13} \mathrm{C}$ NMR Spectrum of Compound 4

${ }^{1} \mathrm{H}$ NMR Spectrum of Compound 8a

${ }^{13} \mathrm{C}$ NMR Spectrum of Compound 8a

${ }^{1} \mathrm{H}$ NMR Spectrum of Compound 8b

${ }^{13} \mathrm{C}$ NMR Spectrum of Compound $8 \mathrm{~b}$

${ }^{1} \mathrm{H}$ NMR Spectrum of Compound 8c

${ }^{13} \mathrm{C}$ NMR Spectrum of Compound 8c

${ }^{1} \mathrm{H}$ NMR Spectrum of Compound 8d

${ }^{13} \mathrm{C}$ NMR Spectrum of Compound 8d

${ }^{1} \mathrm{H}$ NMR Spectrum of Compound 8e

${ }^{13} \mathrm{C}$ NMR Spectrum of Compound 8e

${ }^{1} \mathrm{H}$ NMR Spectrum of Compound $8 \mathrm{f}$

${ }^{13} \mathrm{C}$ NMR Spectrum of Compound $8 \mathrm{f}$

${ }^{1} \mathrm{H}$ NMR Spectrum of Compound 8g

${ }^{13} \mathrm{C}$ NMR Spectrum of Compound $8 \mathrm{~g}$ 


\section{Experimental Section}

General. Aldehydes were obtained from commercial sources. Their purity was checked by ${ }^{1} \mathrm{H}$ NMR and, whenever necessary, purified by distillation or chromatography to ensure no carboxylic acid was present. Allyltributyltin was purchased from Aldrich and used as received. THF was distilled from sodium/benzophenone under a nitrogen atmosphere prior to use. $N, N$ Dimethylformamide (DMF) (anhydrous; Sure-Seal bottle) was purchased from Aldrich. $N, N$ Dimethylacetamide (DMA) and DMSO were dried and freshly distilled over molecular sieves. THF- $d_{8}$ was purchased from Cambridge Chemicals and dried over flamed dried molecular sieves prior to use. Unless otherwise indicated, ${ }^{1} \mathrm{H}$ and ${ }^{13} \mathrm{C}$ NMR spectra were recorded at $25{ }^{\circ} \mathrm{C}$ in $\mathrm{CDCl}_{3}$ at 500 and $125 \mathrm{MHz}$, respectively. Chemical shifts (ppm) were obtained by referencing to $\mathrm{CDCl}_{3}$ as a standard. Flash chromatography was performed on grade 60 silica gel $(32-63 \mu \mathrm{m})$ obtained from commercial sources. All reactions were performed under an argon atmosphere by standard Schlenk techniques. All reactions were performed in duplicate.

Synthesis of the SeCSe-Pd(II) Pincer Complex 4. An oven-dried flask was charged with bis-selenide ligand $5^{11}$ (435 mg, $1.045 \mathrm{mmol}$ ) and $1 \mathrm{~mL}$ of glacial HOAc. Palladium acetate (236 $\mathrm{mg}, 1.05 \mathrm{mmol}$ ) was then added followed by an additional $1 \mathrm{~mL}$ of HOAc. The reaction mixture was heated to $116^{\circ} \mathrm{C}$ and maintained at gentle reflux for $3 \mathrm{~h}$. After the reaction was cooled to $\mathrm{rt}$ and HOAc removed in vacuo, the crude reaction product was dissolved in dichloromethane (2 $\mathrm{mL})$. Hexanes $(10 \mathrm{~mL})$ were then added and the product precipitated as a yellow crystalline solid. The precipitates were allowed to settle and the solvent was decanted. This process was repeated three times and the purified product was dried on vacuum line overnight to give pure 4 (498 mg, 82\%) as a bright yellow solid, m.p. $185-188^{\circ} \mathrm{C}$. Spectroscopic analysis showed that this material exists as a diastereomeric mixture in a ratio of $3: 2 .{ }^{1} \mathrm{H}$ NMR $\left(500 \mathrm{MHz}, \mathrm{CDCl}_{3}\right): \delta$ $7.98(\mathrm{dd}, J=1.86$ and $7.7 \mathrm{~Hz}$, major diastereomer) and 7.91 (d, $J=7.2 \mathrm{~Hz}$, minor diastereomer) 
(4 $\mathrm{H}$ in total), 7.33-7.41 (m, 6H), 6.87-6.94 (m, 3H), 4.573 (minor, $\mathrm{d}, J=14.2 \mathrm{~Hz})$ and $4.568(\mathrm{~d}$, $J=13.7 \mathrm{~Hz}$, major diastereomer) $(2 \mathrm{H}$ in total), $4.32(\mathrm{~d}, J=13.7 \mathrm{~Hz}$, major) and $4.21(\mathrm{~d}, J=14.1$ $\mathrm{Hz}$, minor diastereomer) (2 $\mathrm{H}$ in total), 1.84 (bs, minor diastereomer) and 1.71 (bs, major diastereomer) (3 $\mathrm{H}$ in total). ${ }^{13} \mathrm{C} \mathrm{NMR}\left(125 \mathrm{~Hz}, \mathrm{CDCl}_{3}\right): \delta 177.2$ (minor diastereomer) and 176.9 (major diastereomer), 152.7 (minor diastereomer) and 152.3 (major diastereomer), 150.7 (minor diastereomer) and 150.1 (major diastereomer), 133.3 (major diastereomer) and 133.2 (minor diastereomer), 130.0, 129.8, 129.59, 129.56 and 129.4 (isomeric peaks of $3 \mathrm{C}$ ), 124.5 (major diastereomer) and 124.3 (minor diastereomer), 123.7 (minor diastereomer) and 123.6 (major diastereomer), 43.0 (major diastereomer) and 42.4 (minor diastereomer), 23.6 (minor diastereomer) and 23.5 (major diastereomer). Anal. Calcd. for $\mathrm{C}_{22} \mathrm{H}_{20} \mathrm{O}_{2} \mathrm{PdSe}_{2}: \mathrm{C}, 45.50 ; \mathrm{H}, 3.47$. Found: C, 45.45; H, 3.48.

\section{Allylation of Aldehydes with Allyltributyltin in the Presence of the SeCSe-Pd(II) Pincer}

Catalyst 4. Representative Procedure: Allylation of 4-bromobenzaldehyde (6a) with allyltributyltin (7) in the presence of 4 (Table 1, entry 6). A flame-dried Schlenk flask was charged with aldehyde 6a $(92.5 \mathrm{mg}, 0.5 \mathrm{mmol}), \mathrm{DMF}(0.5 \mathrm{~mL})$ and catalyst $4(2.9 \mathrm{mg}, 0.0050$ mmol) under argon. Allyltributyltin (7) $(186 \mu \mathrm{L}, 199 \mathrm{mg}, 0.6 \mathrm{mmol})$ was then added via syringe. The flask was sealed and heated to $40{ }^{\circ} \mathrm{C}$ (bath temperature) for $18 \mathrm{~h}$. After cooling to rt, the reaction mixture was poured into water and extracted with ether. The combined extracts were stirred with aqueous $\mathrm{KF}(10 \%, \mathrm{w} / \mathrm{v})$ overnight. The organic layer was then separated, washed with brine, dried $\left(\mathrm{Na}_{2} \mathrm{SO}_{4}\right)$ and concentrated. The crude product was purified by flash column chromatography (hexanes/EtOAc 8:1) to give the known homoallyl alcohol 8a ${ }^{1}$ (106 $\mathrm{mg}, 93 \%$ ) as a colorless oil. The reaction was repeated one more time and the product was isolated in $95 \%$ yield. ${ }^{1} \mathrm{H}$ NMR (200 MHz): $\delta 7.48$ (d, $2 \mathrm{H}, J=8.5 \mathrm{~Hz}$ ), 7.22 (d, $\left.2 \mathrm{H} J=8.3 \mathrm{~Hz}\right), 5.68-5.88$ (m, 1 
H), 5.10-5.21 (m, $2 \mathrm{H}), 4.67(\mathrm{t}, 1 \mathrm{H}, J=6.4 \mathrm{~Hz}), 2.64$ (bs, $1 \mathrm{H}$, alcohol), $2.44-2.51(\mathrm{~m}, 2 \mathrm{H}) .{ }^{13} \mathrm{C}$ NMR (50 MHz): $\delta$ 142.84, 133.97, 131.43, 127.61, 121.21, 118.69, 72.65, 43.69.

Reactions (each in duplicate) of other aldehydes under a variety of conditions were performed according to the general procedure with appropriate adjustment of the solvent used and the reaction time and temperature. The results were given in Table S-1.

TABLE S-1. Allylation of Aldehydes with Allyltributyltin 7 Using the Pd-Complex 4 as Catalyst

\begin{tabular}{|c|c|c|c|c|c|c|c|}
\hline entry & aldehyde & & $\mathrm{mmol}$ & $\mathrm{mol} \% 4$ & Solvent & conditions & yield $^{b, c}$ \\
\hline 1 & & & 1.0 & 1 & THF (1 mL) & rt (18h) & 86.5 \\
\hline 2 & $-\mathrm{CHO}$ & $6 a$ & 0.5 & 1 & THF $((0.5 \mathrm{~mL})$ & rt (24h) & 92.5 \\
\hline 3 & & & 1.0 & 1 & THF $(1 \mathrm{~mL})$ & $40^{\circ} \mathrm{C}$ (18h) & 67.5 \\
\hline 4 & & & 0.5 & 1 & THF (0.5 mL) & $60^{\circ} \mathrm{C}(18 \mathrm{~h})$ & 64 \\
\hline 5 & & & 0.5 & 1 & DMF (0.5 mL) & rt (18h) & 77 \\
\hline 6 & & & 0.5 & 1 & DMF (0.5 mL) & $40^{\circ} \mathrm{C}$ (18h) & 94 \\
\hline 7 & & & 0.5 & 1 & DMF (0.5 mL) & $60{ }^{\circ} \mathrm{C}(18 \mathrm{~h})$ & 96 \\
\hline 8 & & & 0.5 & 1 & DMSO (0.5 mL) & rt (18h) & 86.5 \\
\hline 9 & & & 0.5 & 1 & DMSO (0.5 mL) & $40^{\circ} \mathrm{C}(18 \mathrm{~h})$ & 96.5 \\
\hline 10 & & & 0.5 & 1 & DMSO (0.5 mL) & $60^{\circ} \mathrm{C}(18 \mathrm{~h})$ & 97 \\
\hline 11 & & & 1.0 & 1 & DMF (1 mL) & rt (36h) & 87.5 \\
\hline 12 & & 6b & 1.0 & 1 & DMF (1 mL) & $40^{\circ} \mathrm{C}(2 \mathrm{~h})$ & 66.5 \\
\hline 13 & & & 1.0 & 1 & DMF (1 mL) & $40{ }^{\circ} \mathrm{C}(4 \mathrm{~h})$ & 77.5 \\
\hline 14 & & & 1.0 & 1 & DMF (1 mL) & $40{ }^{\circ} \mathrm{C}(6 \mathrm{~h})$ & 87 \\
\hline 15 & & & 0.5 & 1 & DMF (0.5 mL) & $40{ }^{\circ} \mathrm{C}(18 \mathrm{~h})$ & 92.5 \\
\hline 16 & & & 2.0 & 0.25 & DMF $(1 \mathrm{~mL})$ & $40{ }^{\circ} \mathrm{C}(24 \mathrm{~h})$ & 92 \\
\hline 17 & & & 2.0 & 0.02 & DMF (1 mL) & $40{ }^{\circ} \mathrm{C}(40 \mathrm{~h})$ & 95.5 \\
\hline 18 & & & 1.0 & $1(\mathbf{1})^{d}$ & DMF (1 mL) & $40^{\circ} \mathrm{C}(18 \mathrm{~h})$ & 54 \\
\hline 19 & & & $\begin{array}{l}1.0 \\
1.0\end{array}$ & none & DMF (0.5 mL) & $40^{\circ} \mathrm{C}(18 \mathrm{~h})$ & $<1^{\dagger}$ \\
\hline 20 & & & 1.0 & $1(2)^{g}$ & DMF (1 mL) & $40^{\circ} \mathrm{C}(18 \mathrm{~h})$ & 74 \\
\hline 21 & & & 0.5 & 1 & DMF (0.5 mL) & $60^{\circ} \mathrm{C}(18 \mathrm{~h})$ & 58.5 \\
\hline 22 & $-\mathrm{CHO}$ & 6c & 0.5 & 1 & DMSO (0.5 mL) & $60{ }^{\circ} \mathrm{C}(18 \mathrm{~h})$ & 22.5 \\
\hline 23 & & & 2.0 & 1 & DMA (1 mL) & $60^{\circ} \mathrm{C}(18 \mathrm{~h})$ & 64.5 \\
\hline 24 & & & 1.0 & 1 & DMF (0.5 mL) & rt (24h) & 95 \\
\hline 25 & $-\mathrm{CHO}$ & $6 d$ & 1.0 & 1 & DMF (0.5 mL) & $40^{\circ} \mathrm{C}(6 \mathrm{~h})$ & 95.5 \\
\hline 26 & & & 2.0 & 0.25 & DMF $(1 \mathrm{~mL})$ & $40^{\circ} \mathrm{C}(18 \mathrm{~h})$ & 95.5 \\
\hline 27 & & & 4.0 & 0.1 & DMF (2 mL) & $40^{\circ} \mathrm{C}(40 \mathrm{~h})$ & 95.5 \\
\hline 28 & & & 2.0 & 0.02 & DMF (1 mL) & $40^{\circ} \mathrm{C}(24 \mathrm{~h})$ & 95.5 \\
\hline 29 & & & 2.0 & 0.005 & $\mathrm{DMF}(2 \mathrm{~mL})$ & $40^{\circ} \mathrm{C}(40 \mathrm{~h})$ & 73.5 \\
\hline 30 & & & 2.0 & 0.005 & $\mathrm{DMF}(2 \mathrm{~mL})$ & $40^{\circ} \mathrm{C}(96 \mathrm{~h})$ & 79.5 \\
\hline 31 & & & 1.0 & 1 & DMF (0.5 mL) & rt (24h) & 94.5 \\
\hline 32 & & $6 e$ & 4.0 & 0.1 & DMF (2 mL) & $40^{\circ} \mathrm{C}(40 \mathrm{~h})$ & 95.5 \\
\hline 33 & $\mathrm{PhCH}_{2} \mathrm{CH}_{2} \mathrm{CHO}$ & $6 f$ & 1.0 & 1 & DMF (0.5 mL) & $40^{\circ} \mathrm{C}(40 \mathrm{~h})$ & 66.5 \\
\hline 34 & $\mathrm{CH}_{3}\left(\mathrm{CH}_{2}\right)_{5} \mathrm{CH}_{2} \mathrm{CHO}$ & $6 \mathrm{~g}$ & 1.0 & 1 & DMF (0.5 mL) & $40{ }^{\circ} \mathrm{C}(18 \mathrm{~h})$ & 47 \\
\hline 35 & & & 2.0 & 1 & DMF (1 mL) & $60{ }^{\circ} \mathrm{C}(24 \mathrm{~h})$ & 59 \\
\hline
\end{tabular}

${ }^{a}$ All reactions were performed with 1 eq of 6 and 1.2 eq of 7 under conditions as indicated. ${ }^{b}$ Referred to isolated yield after column chromatography on silica gel. ${ }^{c}$ Average of two runs. ${ }^{d}$ The SeCSe-Pd(II) pincer complex 1 was used. ${ }^{e}$ This control reaction was performed in the absence of any catalyst. ${ }^{f}$ Not detected by ${ }^{1}$ H NMR. ${ }^{g}$ The Se-palladacycle 2 was used. 


\section{${ }^{1} \mathrm{H}$ NMR Spectroscopic Study on the Reaction of the Pd Complex 4 with Allyltributyltin} 7: Identification of the Transmetalation Product 9. An NMR tube (5 mm) was charged with 4 (4.6 mg, $0.008 \mathrm{mmol}$ ) and $600 \mu \mathrm{L}$ of degassed THF- $\mathrm{d}_{8}$ was added under an Ar atmosphere. The NMR tube was cooled to $-5^{\circ} \mathrm{C}$ and ${ }^{1} \mathrm{H}$ NMR spectrum (at $500 \mathrm{MHz}$ with $\mathrm{d} 1=10 \mathrm{~s}$ ) was recorded. Due to the chiral nature of the Pd-bound selenium, there exist two diastereomers, cis- and trans-4 (Scheme S-1) as evidenced by the two diastereotopic $\mathrm{SeCH}_{2}$ groups corresponding to each of the diastereomers. Each of the $\mathrm{SeCH}_{2}$ groups appears as two doublets (Figure S-1). Allyltributyltin $7(24.5 \mu \mathrm{L}, 26.5 \mathrm{mg}, 0.080 \mathrm{mmol})$ was then added and the temperature of the NMR probe was raised to $25{ }^{\circ} \mathrm{C}$. After $20 \mathrm{~min}$ at this temperature, 4 was converted to a new compound in about $50 \%$ conversion. This new compound was identified as the transmetalation product $\mathbf{9}-\eta^{1}$. The ${ }^{1} \mathrm{H}$ NMR spectrum of the reaction mixture at $-5^{\circ} \mathrm{C}$ was shown in Figure S-2. The spectrum assignments for compounds 4, 7 and 9 were shown in Figure S-3. The 2D proton COSY NMR spectra of the reaction mixture were shown in Figures S-4.A and S-4.B.

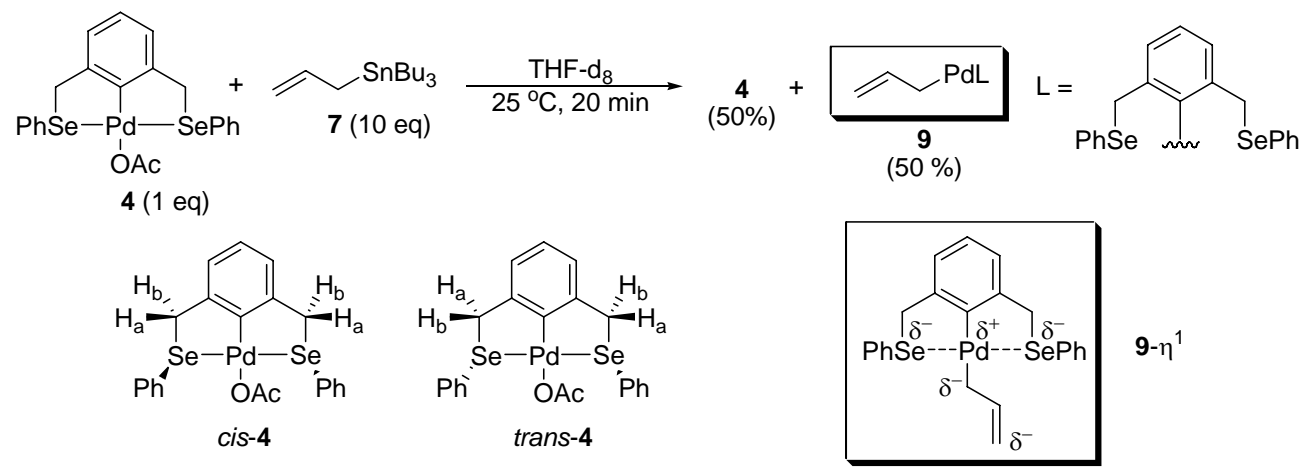

SCHEME S-1. Reaction of Pd Complex 4 with Allyltributyltin 7 in THF- $\mathrm{d}_{8}$. 


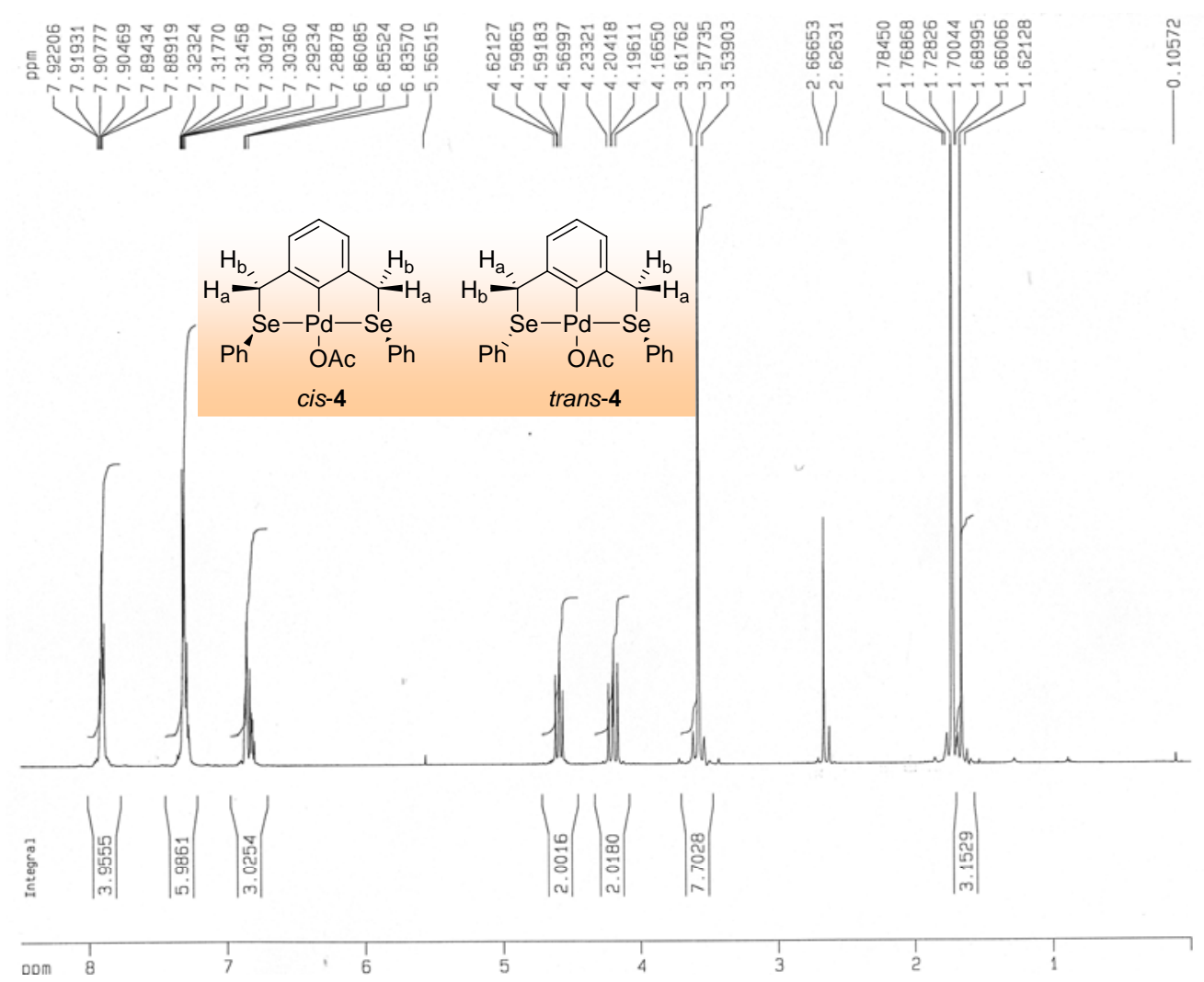

Figure S-1. ${ }^{1} \mathrm{H}$ NMR Spectrum of 4 in THF- $\mathrm{d}_{8}$ to $25^{\circ} \mathrm{C}$.

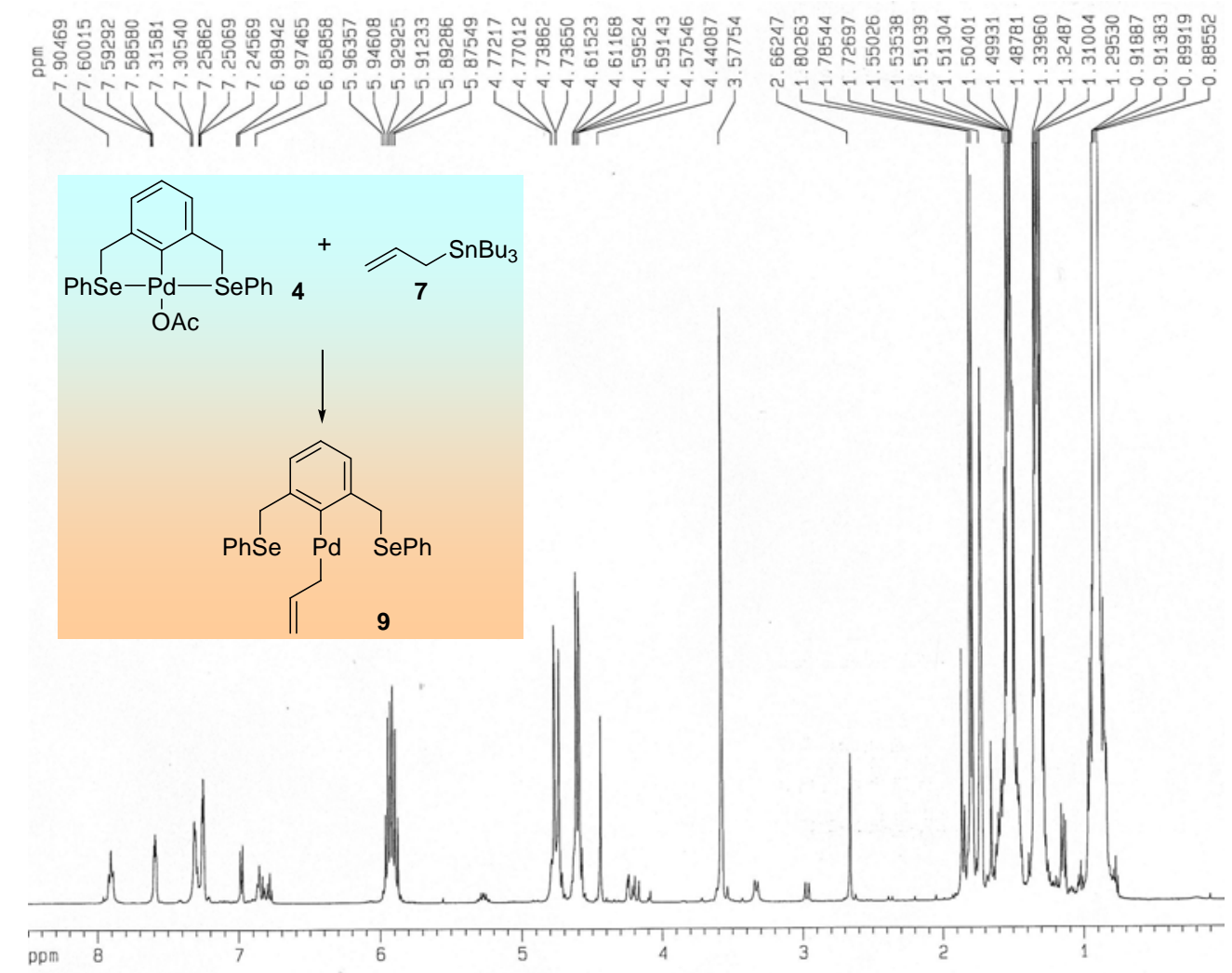

FiguRE S-2. ${ }^{1} \mathrm{H}$ NMR Spectrum of the Reaction Mixture of 4 and 7. 


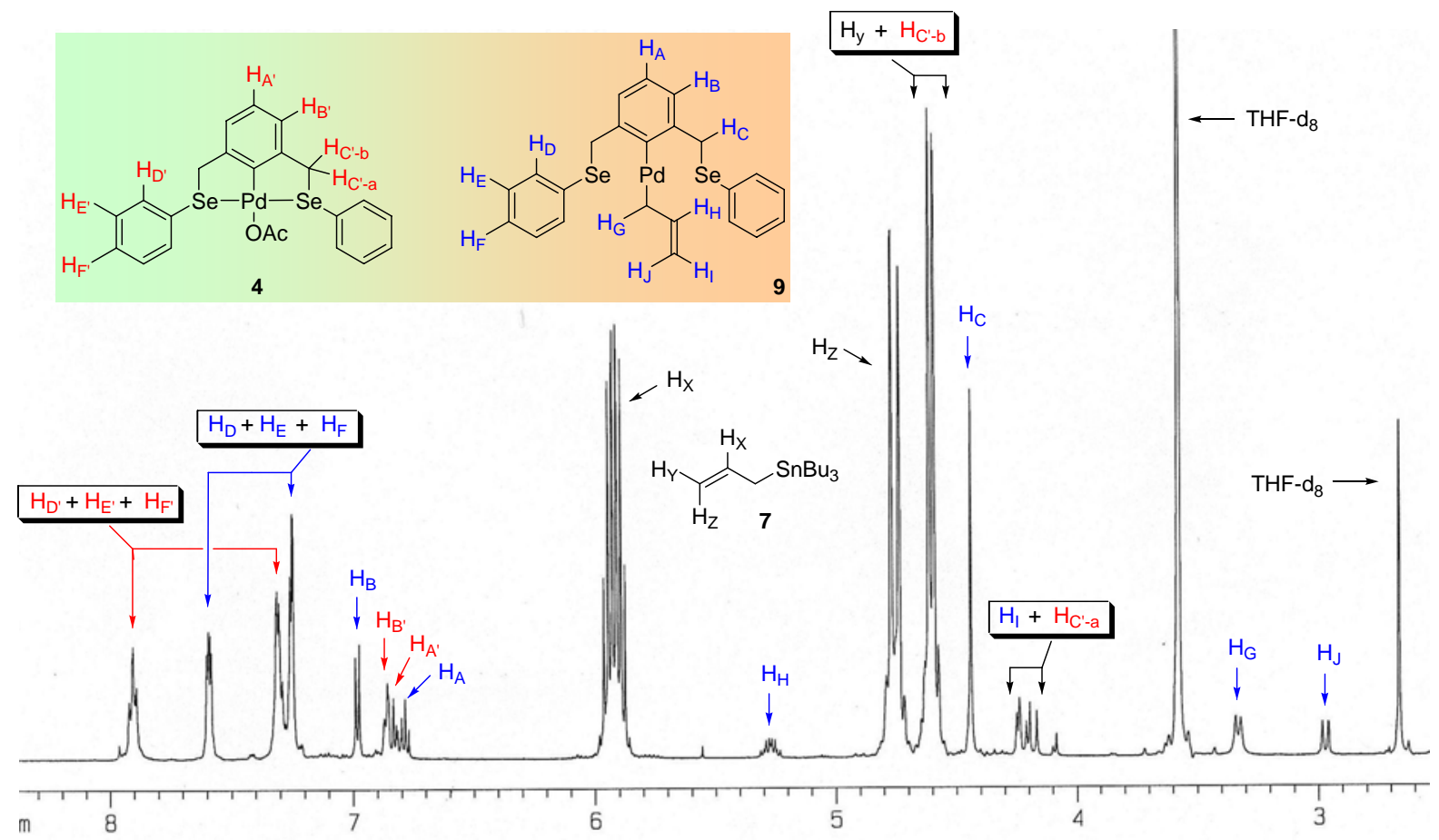

FIgURE S-3. Partial $500 \mathrm{MHz}{ }^{1} \mathrm{H}$ NMR Spectrum and Spectrum Assignments for the Crude Reaction Mixture Containing 4, 7 and the Allyl-Pd(II) Complex $9^{14}$ in THF- $\mathrm{d}_{8}$ at $-5^{\circ} \mathrm{C}$.

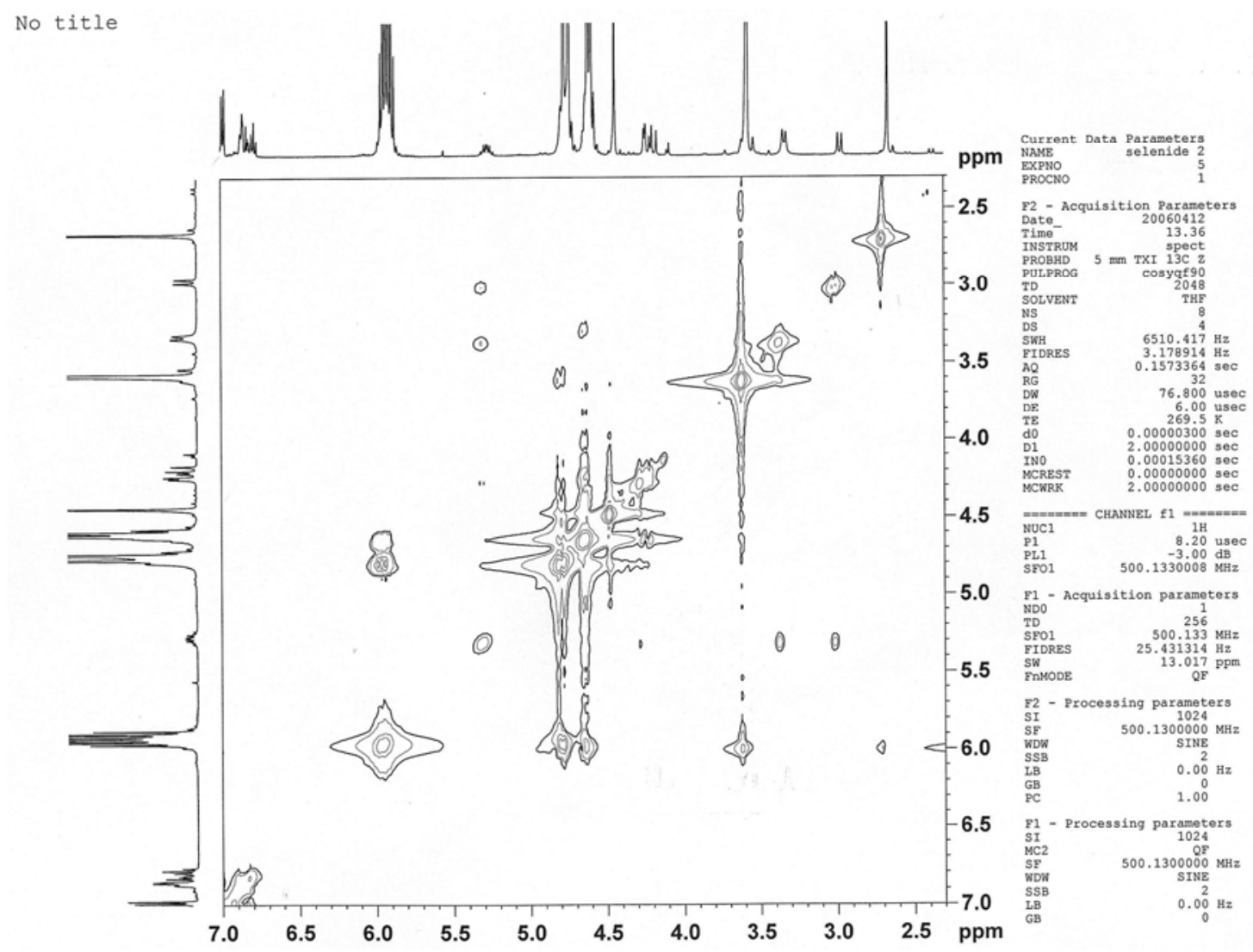

Figure S-4. A. 2D COSY Spectrum (Plot A) of the Crude Reaction Mixture of 4 and 7. 


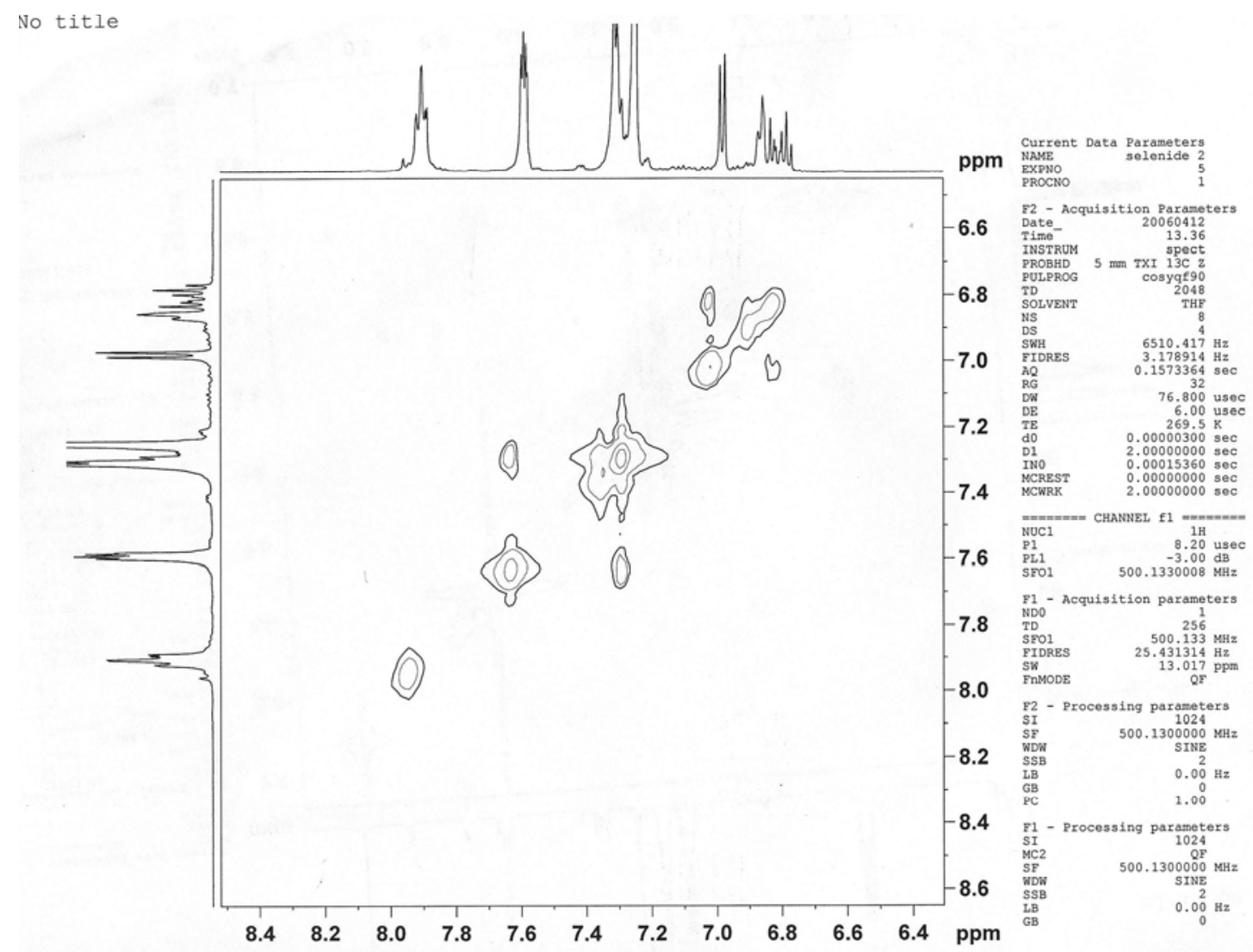

Figure S-4. B. 2D COSY Spectrum (Plot B) of the Reaction Mixture of 4 and 7.

Phenylbut-3-en-1-ol (8b) ${ }^{2}$<smiles>C=CCC(O)c1ccccc1</smiles>

${ }^{1} \mathrm{H}$ NMR: $\delta$ 7.29-7.41 (m, $\left.5 \mathrm{H}\right), 5.79-5.88(\mathrm{~m}, 1 \mathrm{H}), 5.15-5.20(\mathrm{~m}, 2 \mathrm{H}), 4.71(\mathrm{t}, 1 \mathrm{H} J=6.5 \mathrm{~Hz})$, 2.79 (bs, $1 \mathrm{H}$ alcohol), 2.54 (t, $2 \mathrm{H}, J=6.8$ ). ${ }^{13} \mathrm{C}$ NMR: $\delta 144.22,134.74,128.39,127.47$, $126.08,117.85,73.56,43.72$.

\section{1-(4-Methoxyphenyl)but-3-en-1-ol (8c) ${ }^{1,3}$}<smiles>C=CCC(O)c1ccc(OC)cc1</smiles> 
${ }^{1} \mathrm{H}$ NMR: $\delta 7.30$ (d, $\left.2 \mathrm{H}, J=8.5 \mathrm{~Hz}\right), 6.90$ (d, $\left.2 \mathrm{H}, J=8.5 \mathrm{~Hz}\right), 5.78-5.86(\mathrm{~m}, 1 \mathrm{H}), 5.13-5.18$ (m, $2 \mathrm{H}), 4.69$ (t, $1 \mathrm{H}, J=6.5 \mathrm{~Hz}), 3.82(\mathrm{~s}, 3 \mathrm{H}), 2.52(\mathrm{t}, 2 \mathrm{H}, J=7.0 \mathrm{~Hz}), 2.18$ (bs, $1 \mathrm{H}$, alcohol). ${ }^{13} \mathrm{C}$ NMR: $\delta 159.07,136.14,134.65,127.09,118.11,113.64,73.03,55.28,43.64$.

1-(4-Nitrophenyl)but-3-en-1-ol (8d) ${ }^{1 \mathrm{~b}, 3 \mathrm{c}}$<smiles>C=CCC(O)c1ccc([N+](=O)[O-])cc1</smiles>

${ }^{1} \mathrm{H}$ NMR: $\delta 8.14(\mathrm{~d}, 2 \mathrm{H}, J=8.5 \mathrm{~Hz}), 7.52(\mathrm{~d}, 2 \mathrm{H}, J=8.5 \mathrm{~Hz}), 5.74-5.82(\mathrm{~m}, 1 \mathrm{H}), 5.11-5.15$ (m, $2 \mathrm{H}), 4.87$ (t, $1 \mathrm{H}, J=6.0 \mathrm{~Hz}), 3.17$ (bs, 1H, alcohol), 2.45-2.56 (m, $2 \mathrm{H}) .{ }^{13} \mathrm{C}$ NMR: $\delta$ $151.52,147.067,133.36,126.65,123.46,118.96,72.35,43.61$.

4-(1-Hydroxybut-3-enyl)benzonitrile (8e) ${ }^{3 \mathrm{c}}$<smiles>C=CCC(O)c1ccc(C#N)cc1</smiles>

${ }^{1} \mathrm{H}$ NMR: $\delta 7.56(\mathrm{~d}, 2 \mathrm{H}, J=8.0 \mathrm{~Hz}), 7.43(\mathrm{~d}, 2 \mathrm{H}, J=7.5 \mathrm{~Hz}), 5.69-5.77(\mathrm{~m}, 1 \mathrm{H}), 5.07-5.11$ (m, 2H), 4.75 (t, $J=6.1 \mathrm{~Hz}), 3.08$ (bs, 1H, alcohol), 2.39-2.50 (m, 2H). ${ }^{13} \mathrm{C}$ NMR: $\delta$ 149.56, $133.50,132.12,126.61,118.9,110.81,72.48,43.62$.

\section{Phenyl-5-hexen-3-ol (8f)}<smiles>C=CCC(O)CCc1ccccc1</smiles>

${ }^{1} \mathrm{H}$ NMR: $\delta$ 7.20-7.38 (m, 5 H), 5.78-5.90 (m, $\left.1 \mathrm{H}\right), 5.13-5.19$ (m, $\left.2 \mathrm{H}\right), 3.71$ (m, $\left.1 \mathrm{H}\right), 2.67-2.89$ (m, $2 \mathrm{H}), 2.18-2.40$ (m, $2 \mathrm{H}), 1.76-1.86(\mathrm{~m}, 2 \mathrm{H}), 1.66$ (bs, $1 \mathrm{H}$, alcohol). ${ }^{13} \mathrm{C}$ NMR: $\delta 142.07$, $134.63,128.48,128.44,125.87,118.51,69.88,42.12,38.48,32.08$

Undec-1-en-4-ol (8g) ${ }^{1 b, 5}$ 


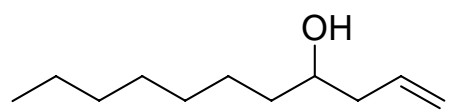

${ }^{1} \mathrm{H}$ NMR: $\delta$ 5.81-5.88 (m, $\left.1 \mathrm{H}\right), 5.02-5.18(\mathrm{~m}, 2 \mathrm{H}), 3.60-3.67(\mathrm{~m}, 1 \mathrm{H}), 2.25-2.29(\mathrm{~m}, 1 \mathrm{H}), 2.20-$ $2.11(\mathrm{~m}, 2 \mathrm{H}), 1.71$ (bs, $1 \mathrm{H}$, alcohol), 1.49-1.28 (m, $12 \mathrm{H}), 0.89$ (t, $1 \mathrm{H}, J=7.0 \mathrm{~Hz}) .{ }^{13} \mathrm{C}$ NMR: $\delta$ 134.94, 117.66, 70.75, 41.91, 36.84, 31.77, 29.58, 29.20, 25.60, 22.56, 13.93.

\section{$\underline{\text { References }}$}

(1) (a) Yanagisawa, A.; Kageyama, H.; Nakatsuka, Y.; Asakawa, K.; Matsumoto, Y.;

Yamamoto, H. Angew. Chem., Int. Ed. 1998, 38, 3701. (b) Thadani, A. N.; Batey, R. A. Org. Lett. 2002, 4, 3827.

(2) (a) Dussault, P. H.; Lee, H.-J.; Liu, X. J. Chem. Soc., Perkin Trans.1 2000, 3006. (b) Cai, M.; Huang, Y.; Zhao, H.; Zhang, R. J. Organomet. Chem. 2004, 689, 2436.

(3) (a) Shibato, A.; Itagaki, Y.; Tayama, E.; Hokke, Y.; Asao, N.; Maruoka, K. Tetrahedron 2000, 56, 5373. (b) Doucet. H.; Santelli, M. Tetrahetron: Asymmetry 2000, 11, 4163. (c)

Fürstner, A.; Voigtlaender, D. Synthesis 2000, 959.

(4) (a) Akiyama, T.; Iwai, J.; Sugano, M. Tetrahedron 1999, 55, 7499. (b) Fujioka, H.; Ohba, Y.; Hirose, H.; Murai, K.; Kita, Y. Org. Lett. 2005, 7, 3303.

(5) Fürstner, A.; Shi, N. J. Am. Chem. Soc. 1996, 118, 12349. 


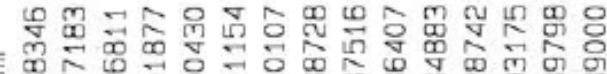

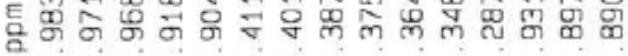

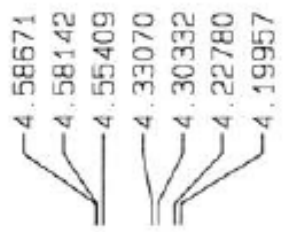

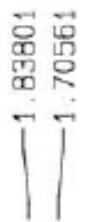
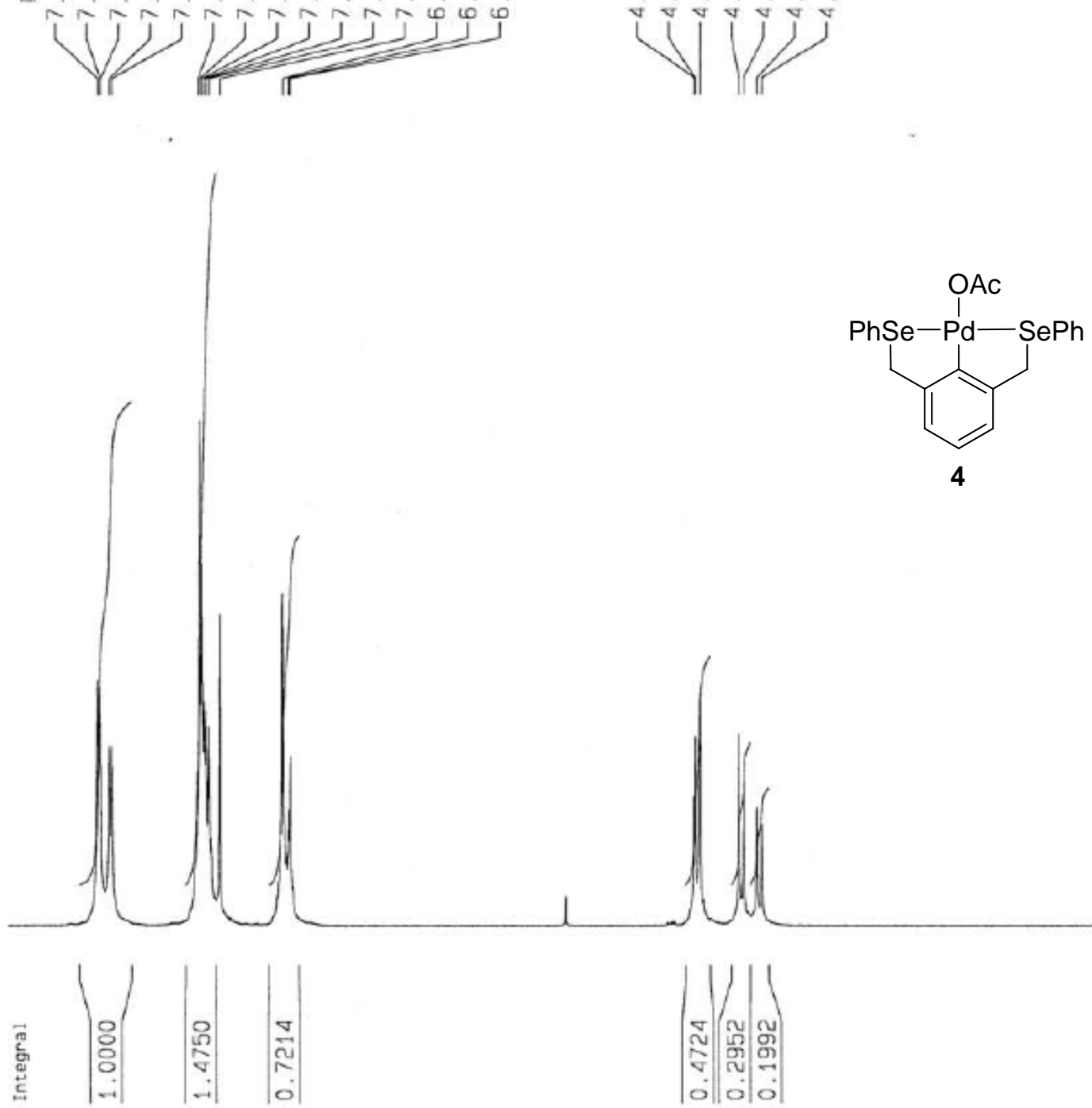

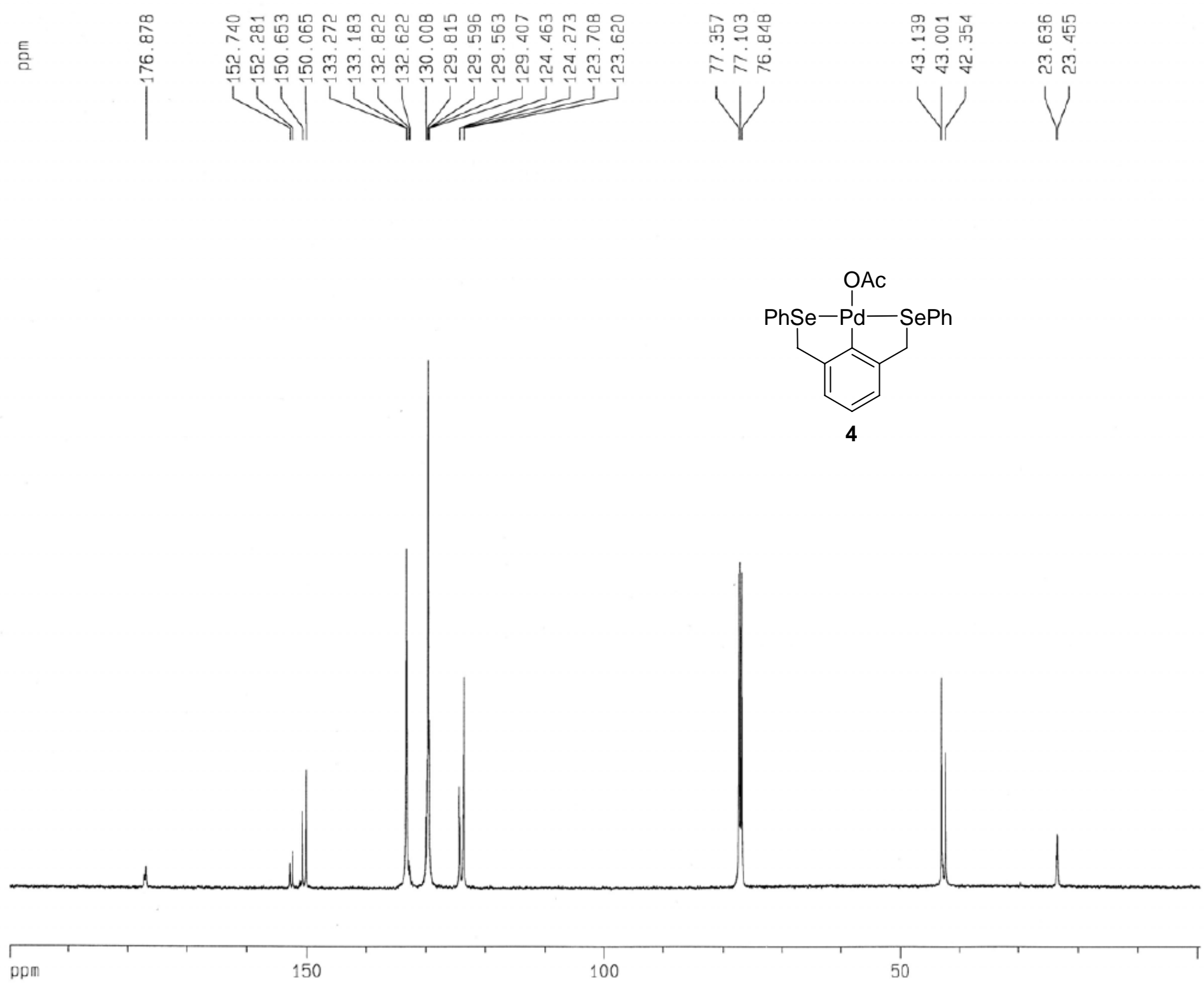

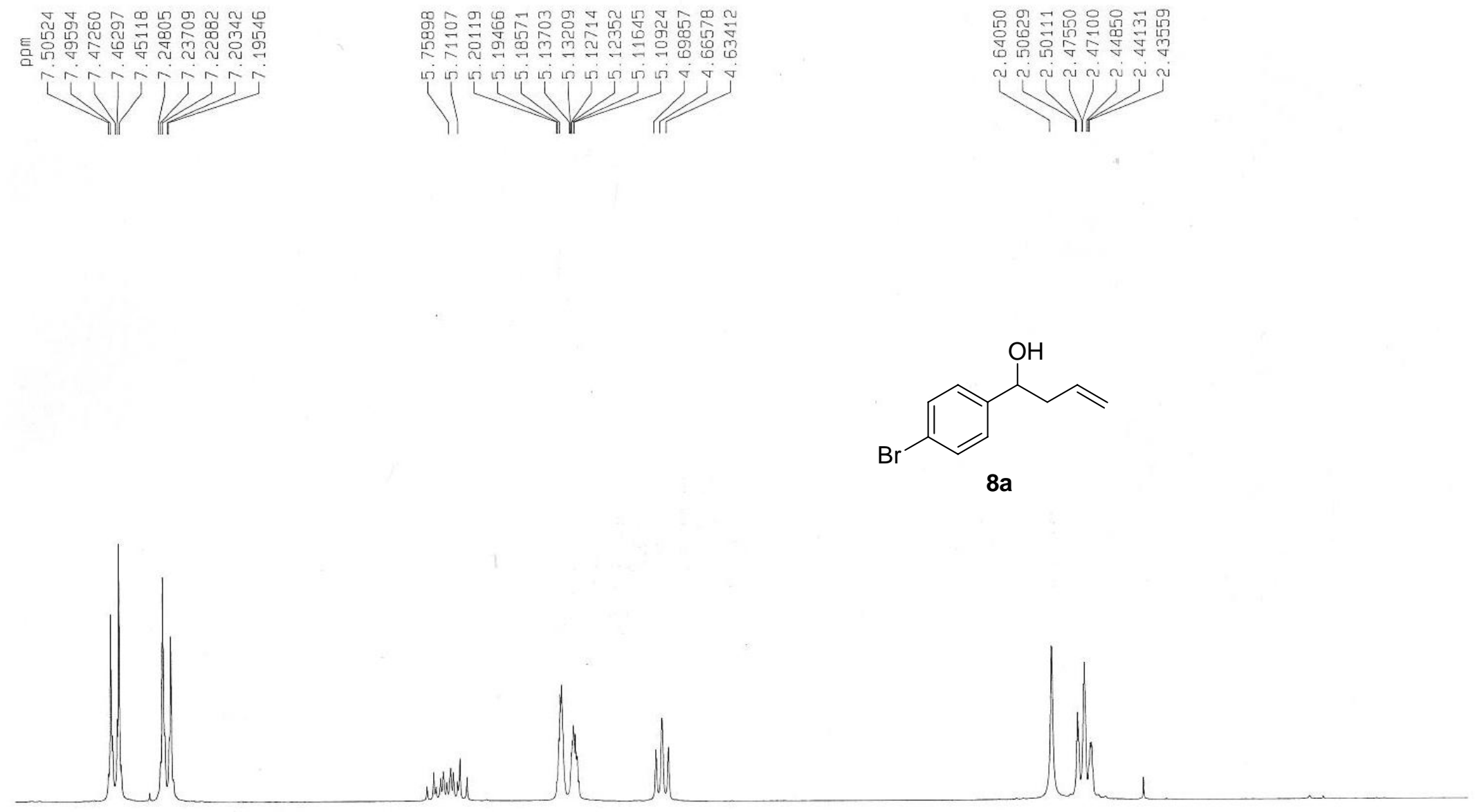

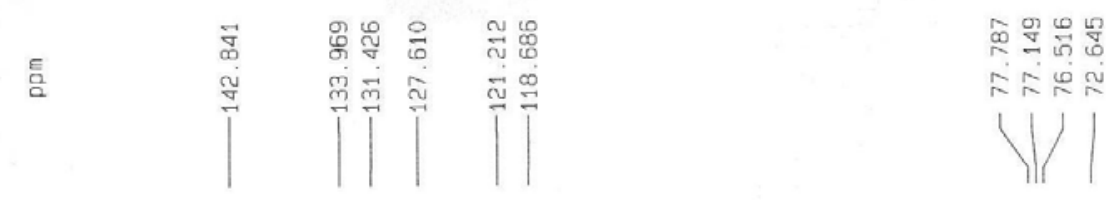

0
0
0
$\dot{0}$
अ
$\mid$

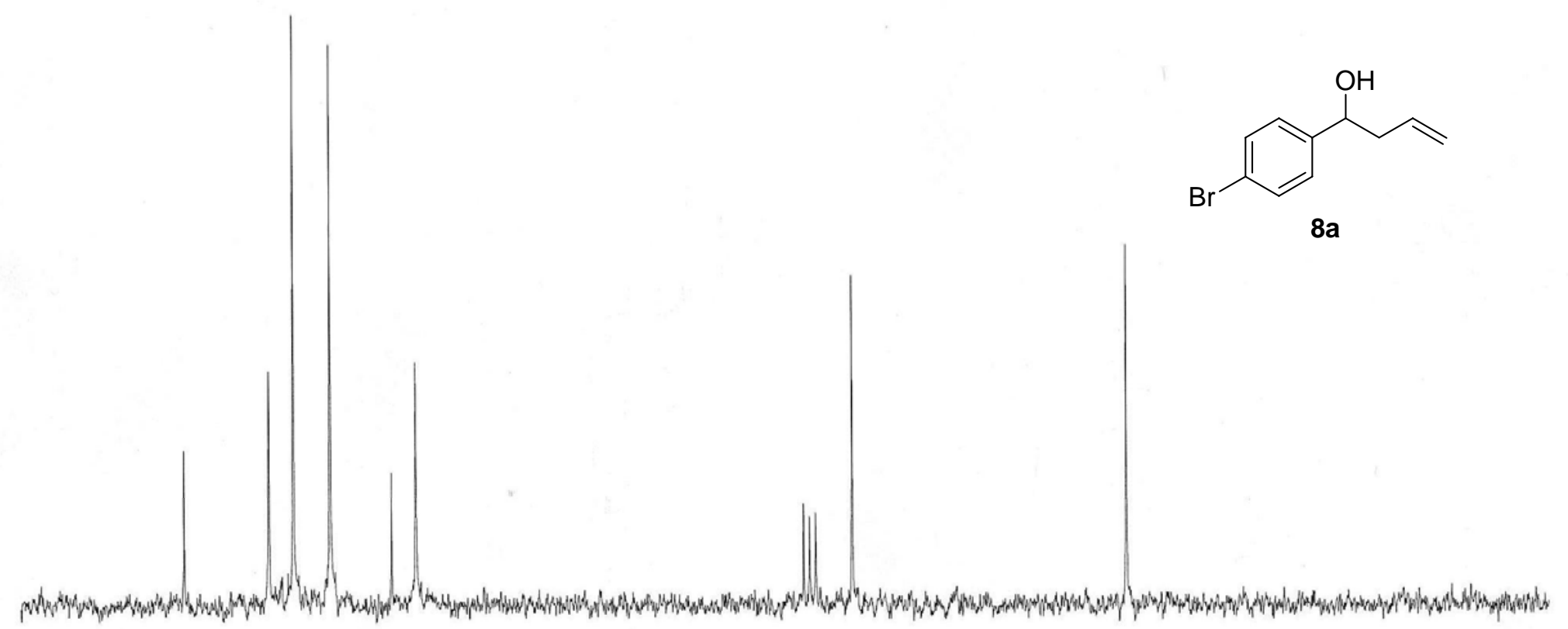



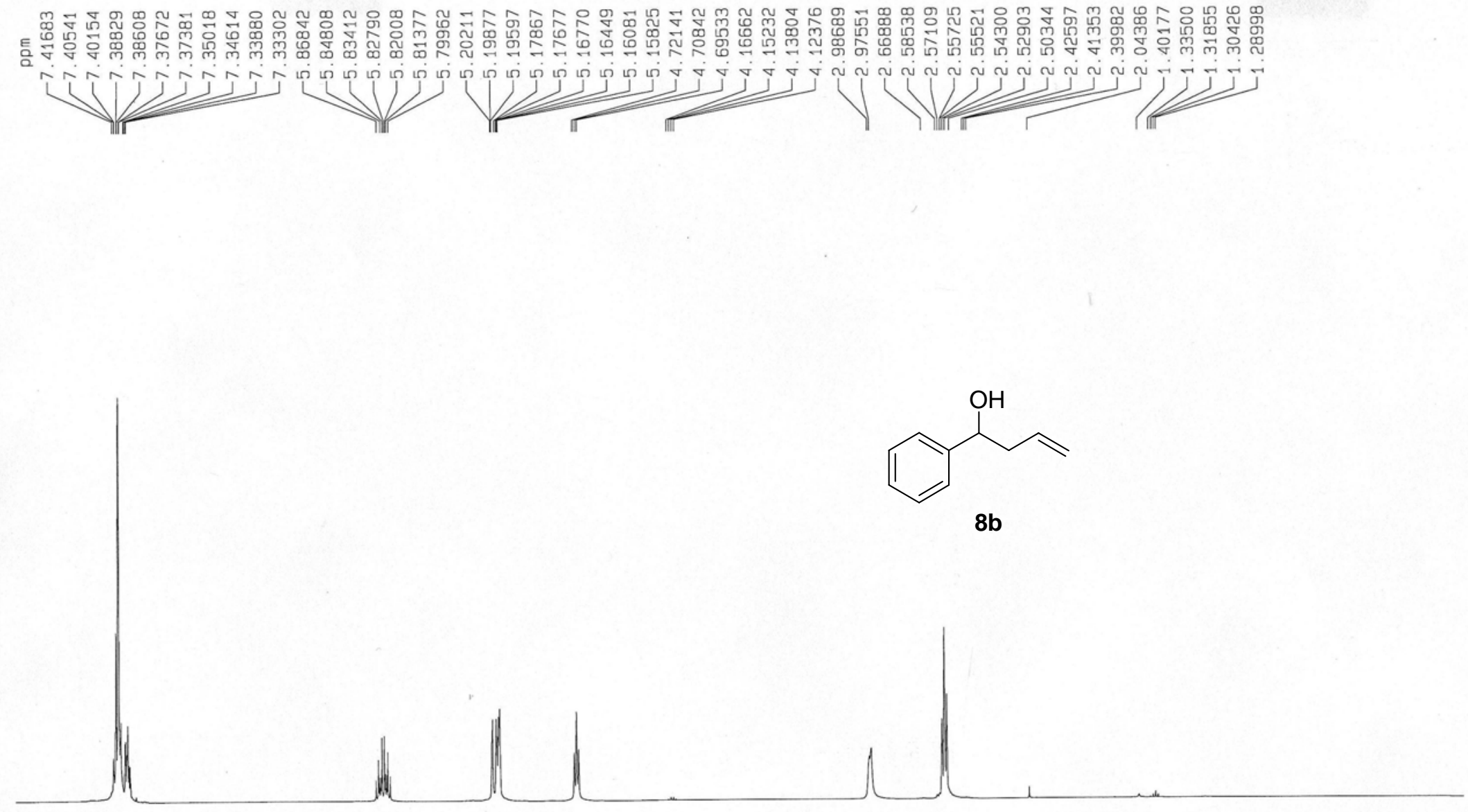


\section{Supporting Information}

틈

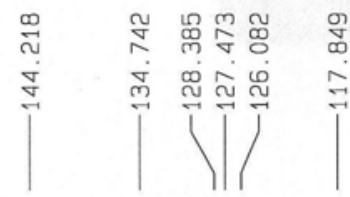

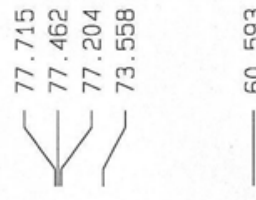

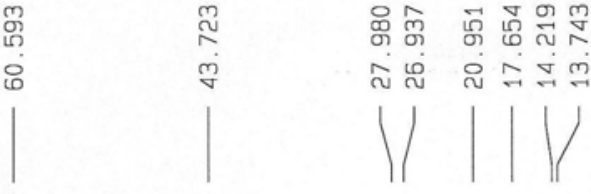

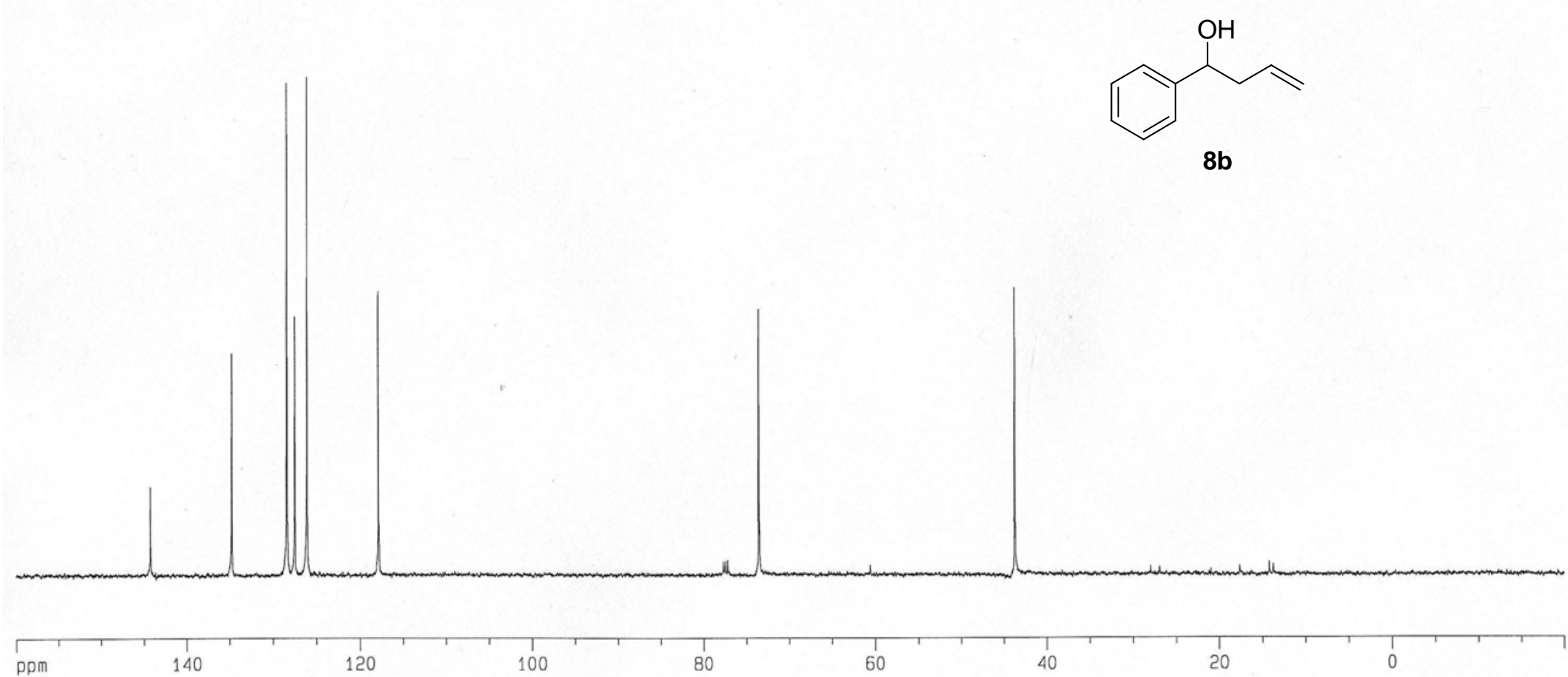




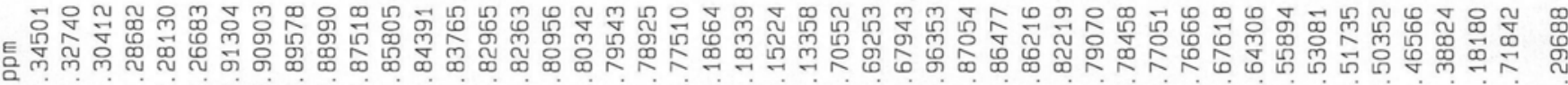
近人
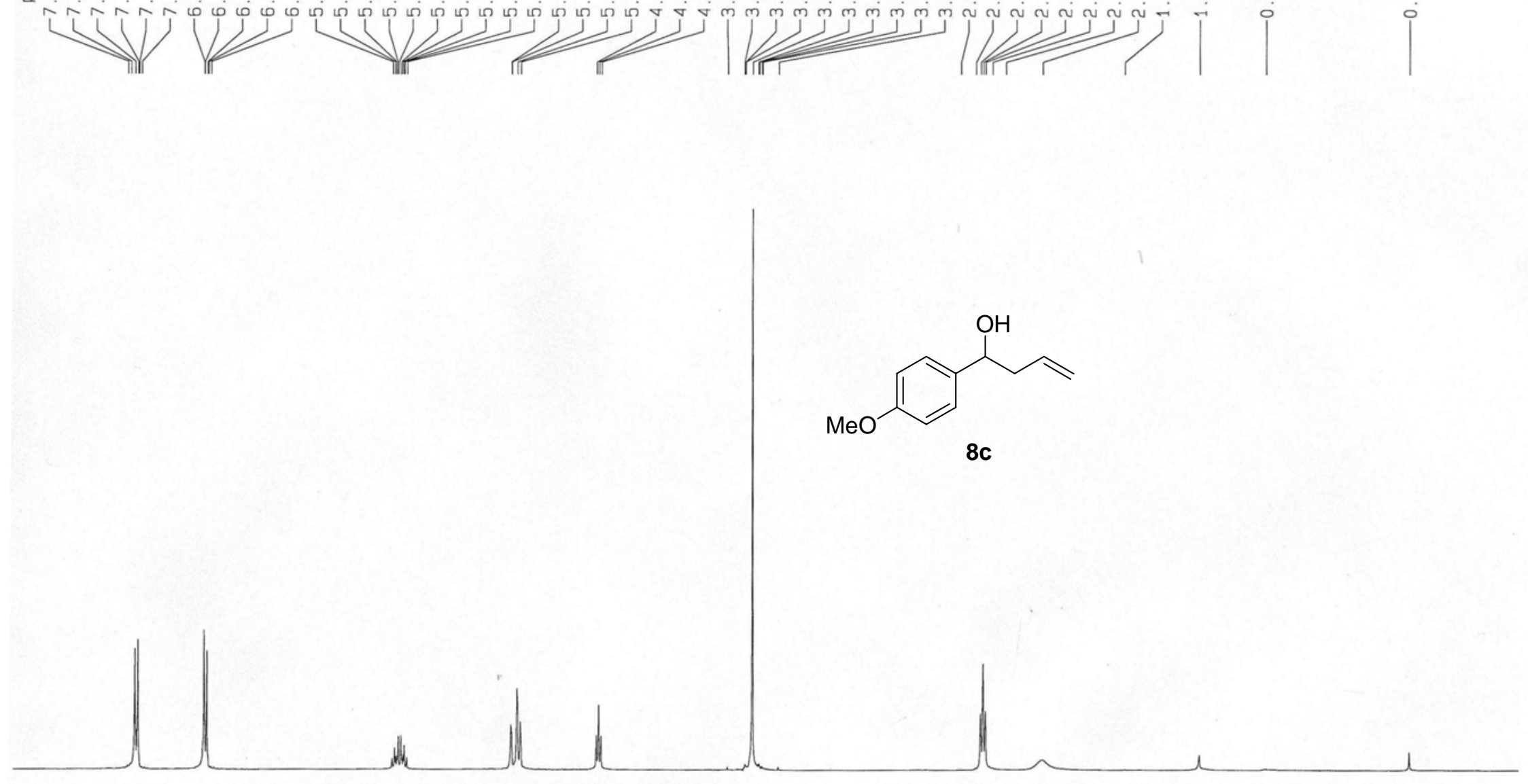


\section{Supporting Information}

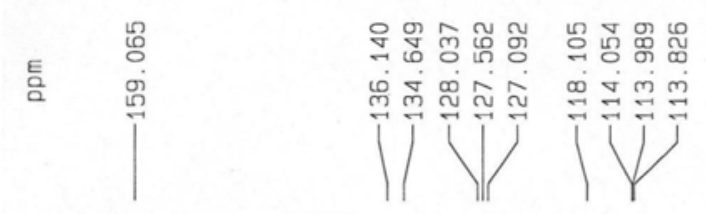

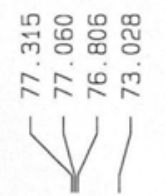

空

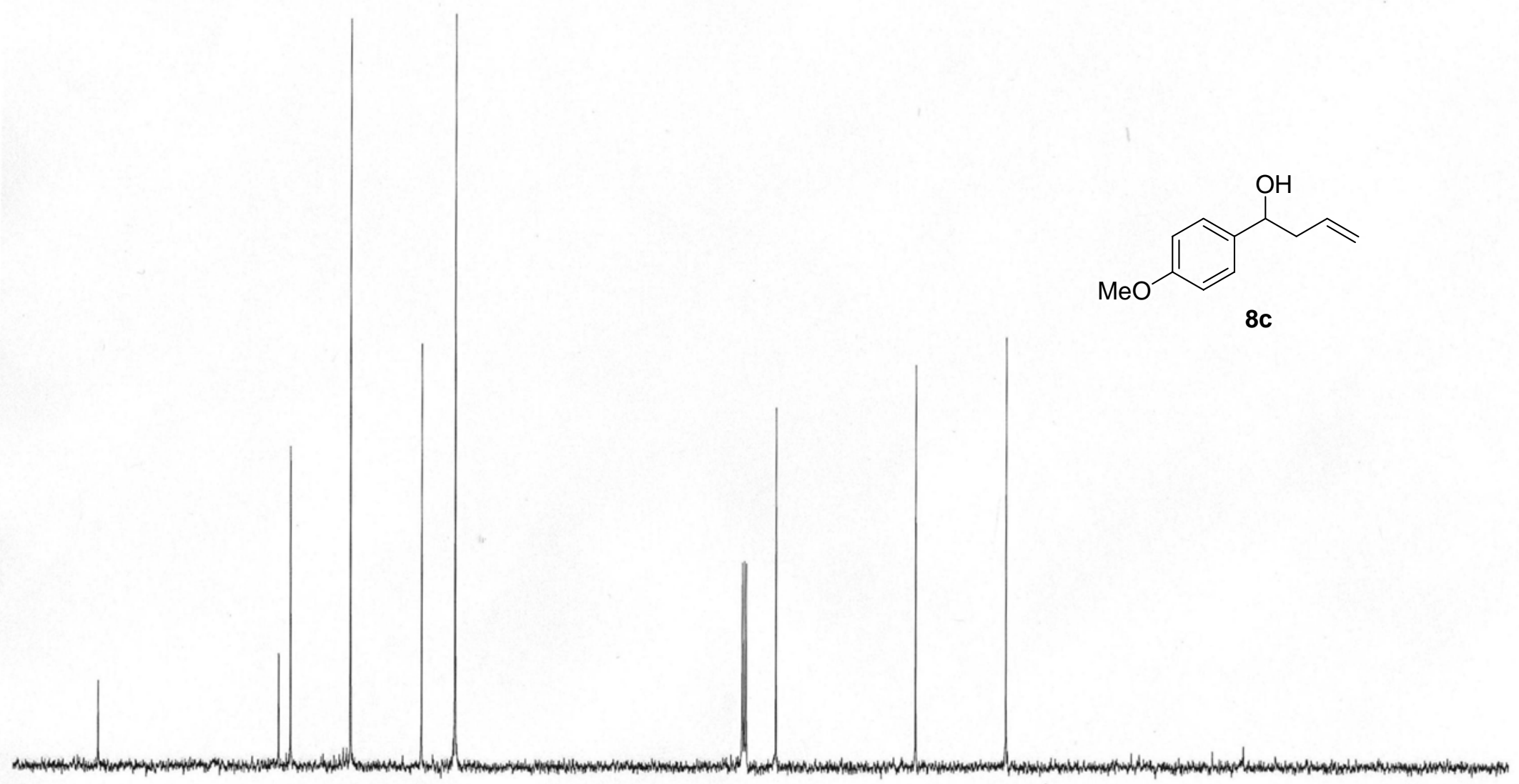




\section{Supporting Information}
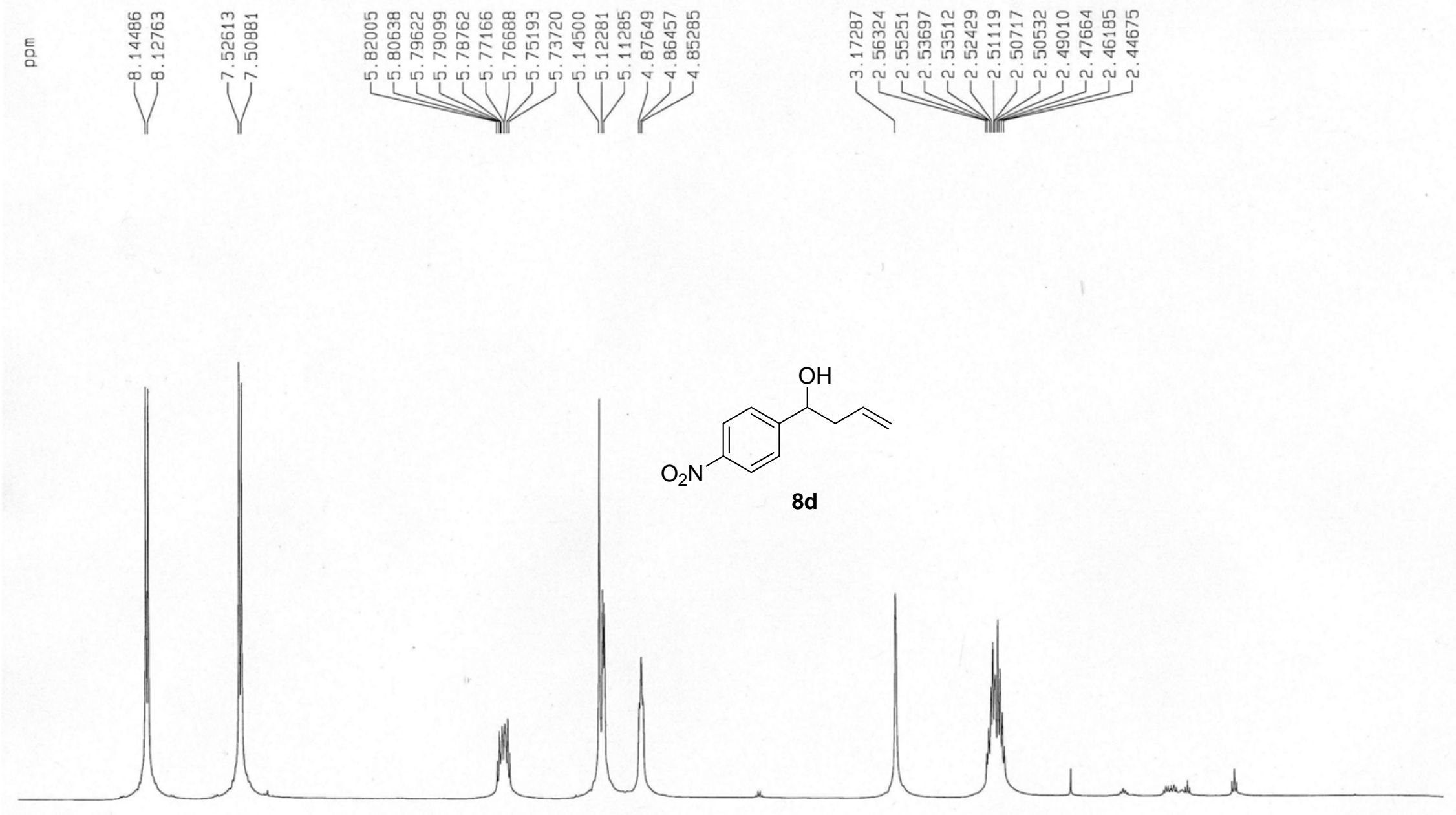


\section{Supporting Information}
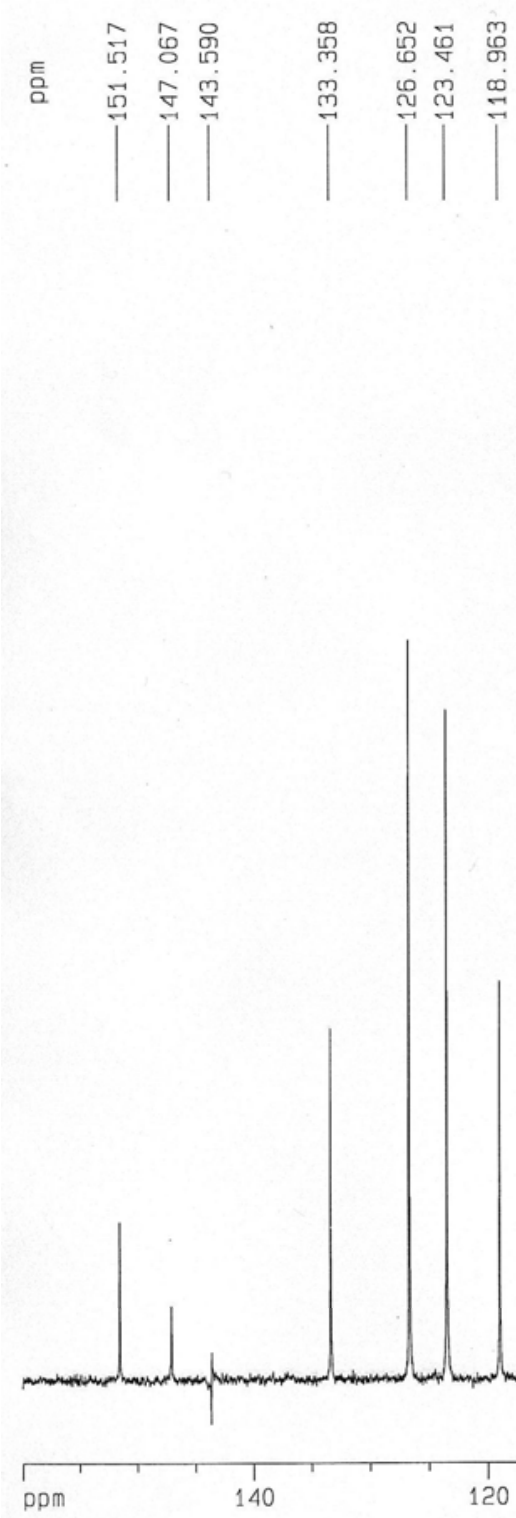

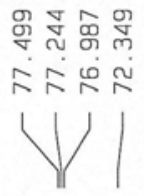

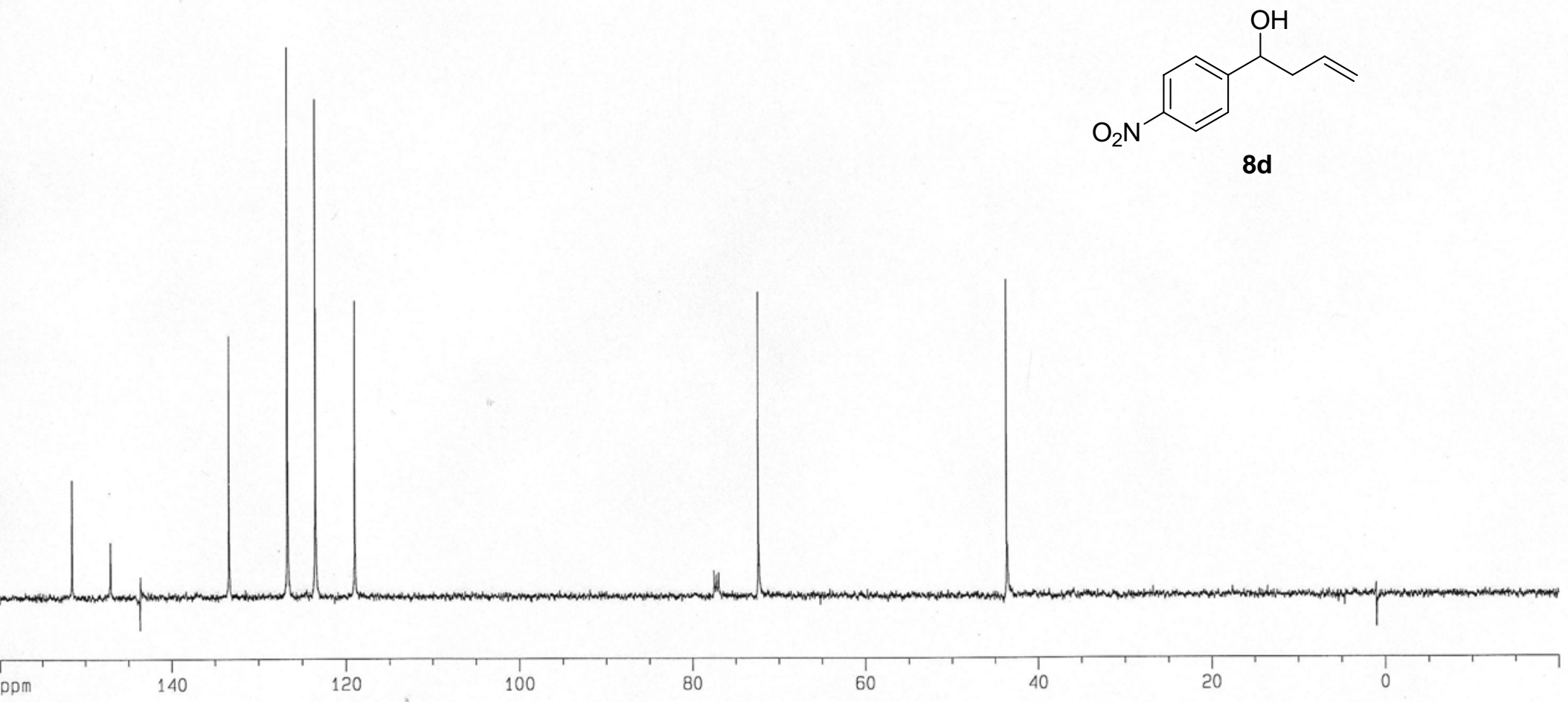



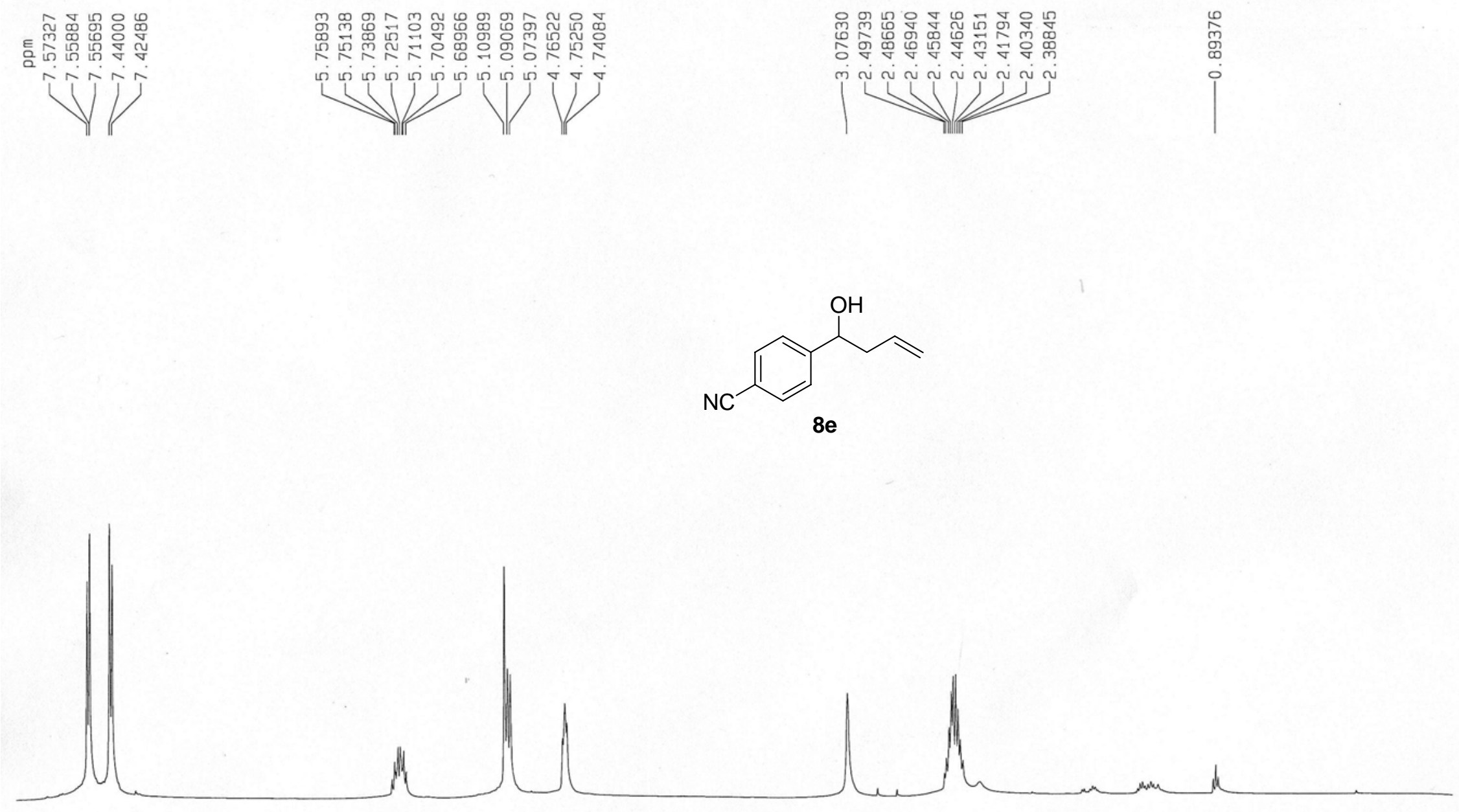


\section{Supporting Information}

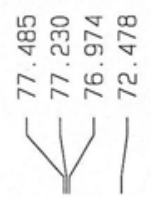

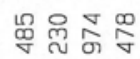

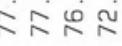

$\checkmark$
ָั
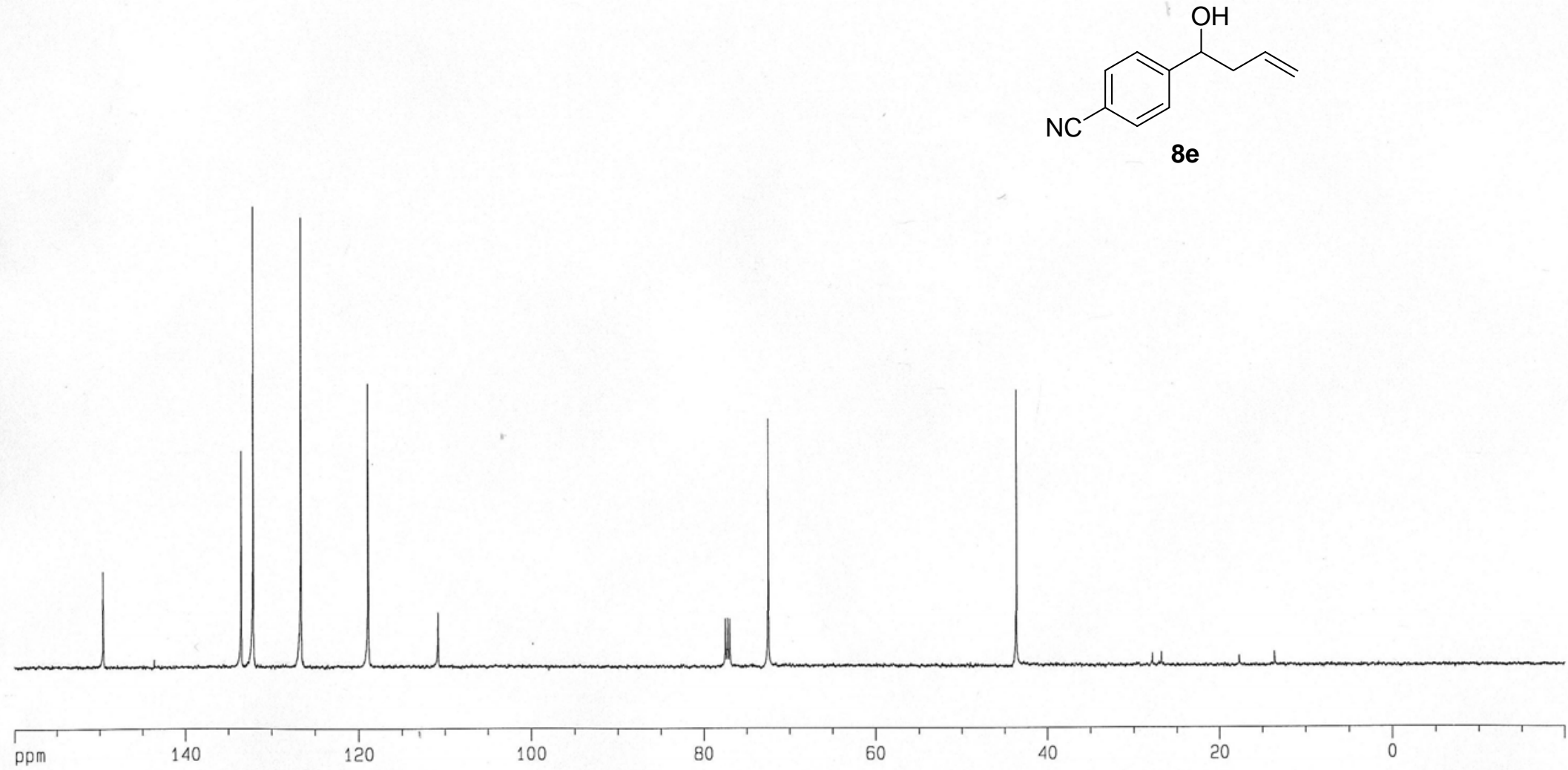


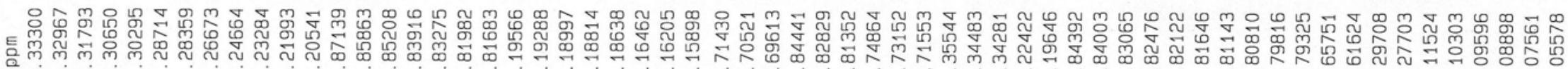

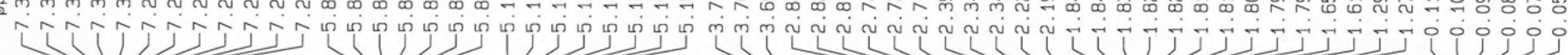
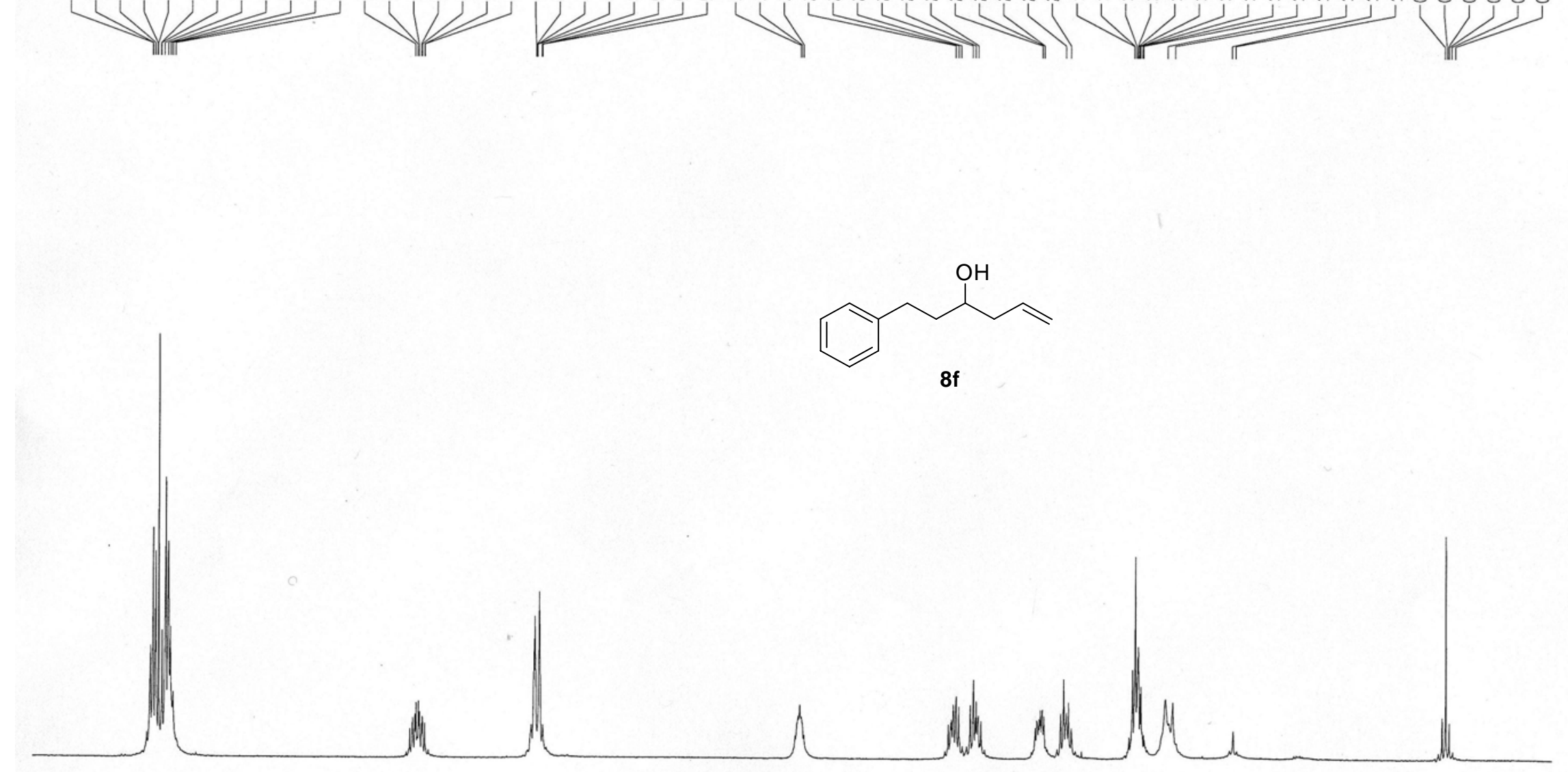


\section{Supporting Information}

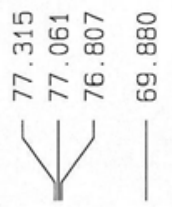

邑诘畣离

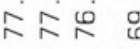

Y

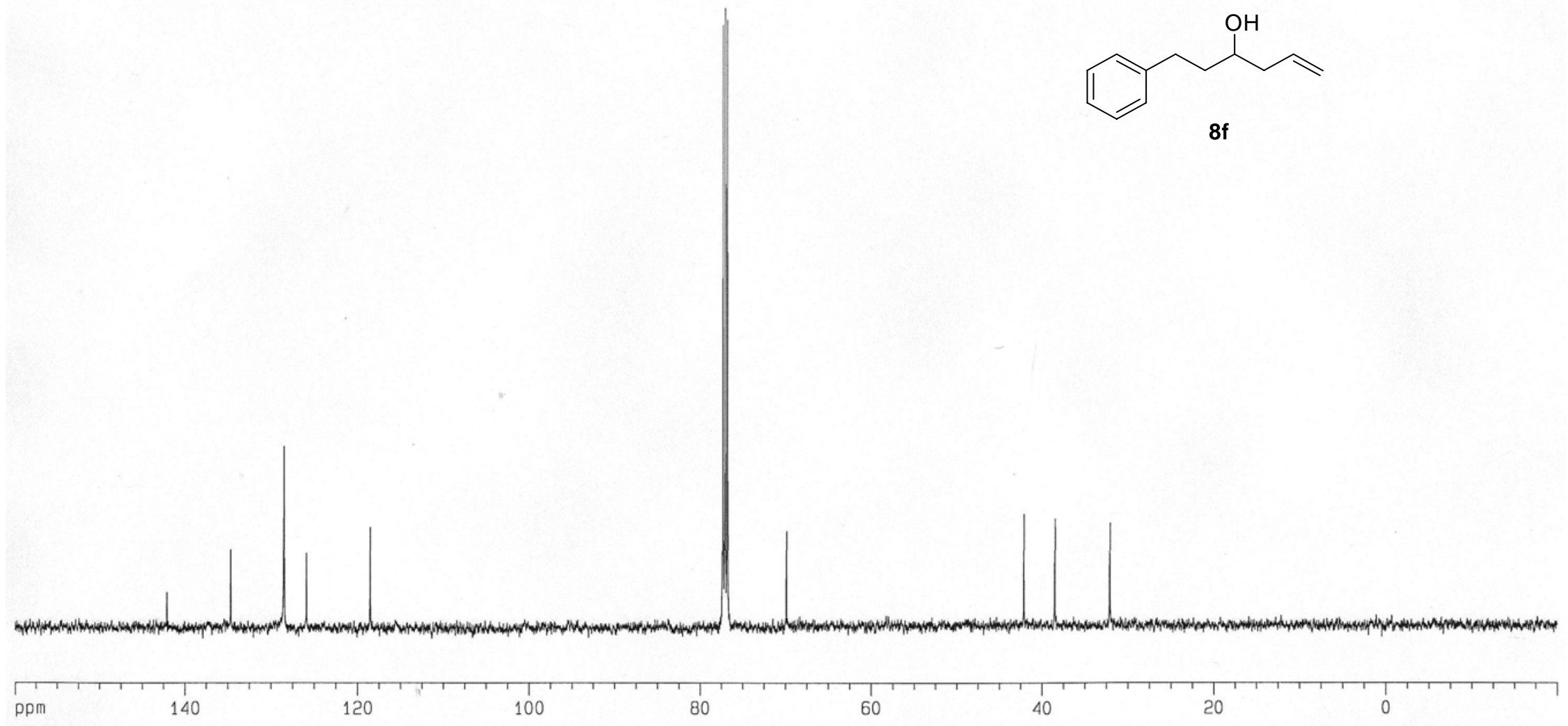

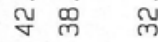

| |

$8 f$ 


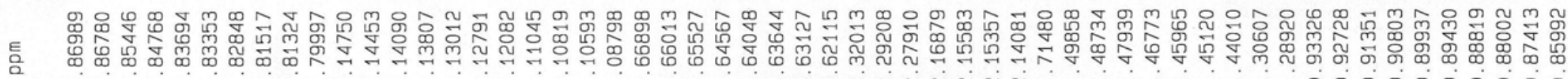

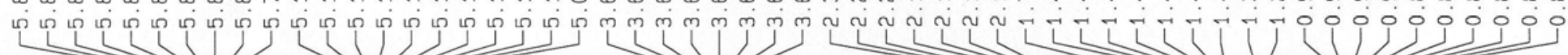

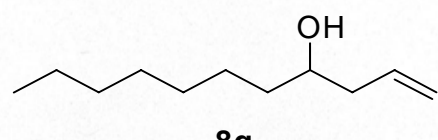

$8 \mathrm{~g}$

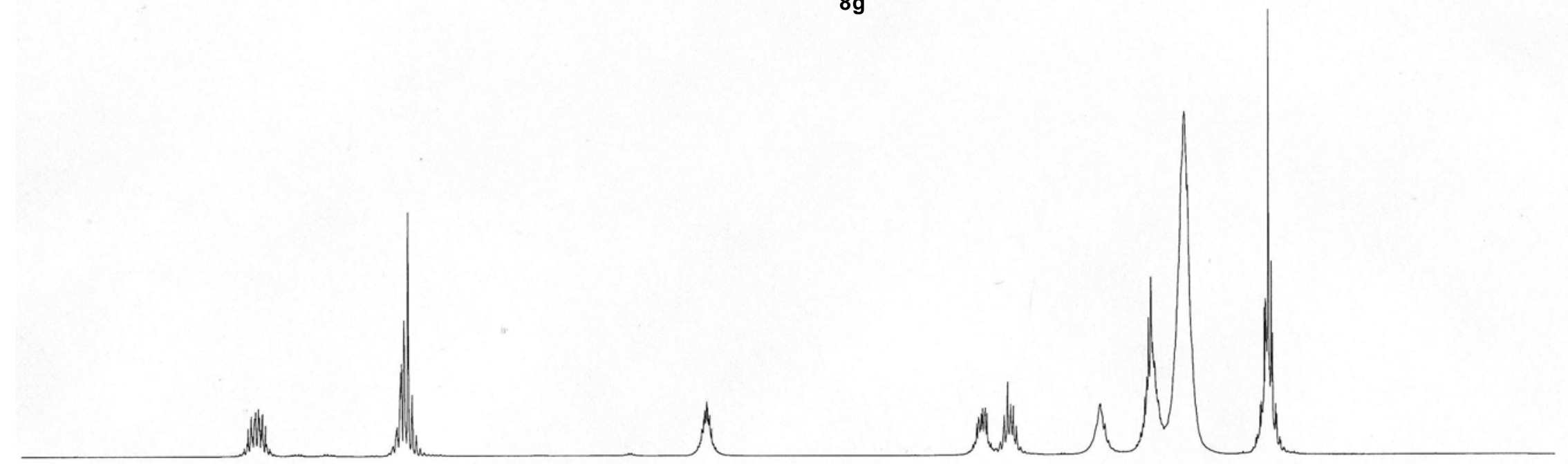


